Article

\title{
Product and Process Fingerprint for Nanosecond Pulsed Laser Ablated Superhydrophobic Surface
}

\author{
Yukui Cai ${ }^{1}{ }^{\circledR}$, Xichun Luo ${ }^{1, *}$, Zhanqiang Liu ${ }^{2,3}{ }^{\circledR}$, Yi Qin ${ }^{1}$, Wenlong Chang ${ }^{1}$ and Yazhou Sun ${ }^{4}$ \\ 1 Centre for Precision Manufacturing, DMEM, University of Strathclyde, Glasgow G1 1XJ, UK; \\ yukui.cai@strath.ac.uk (Y.C.); qin.yi@strath.ac.uk (Y.Q.); wenlong.chang@strath.ac.uk (W.C.) \\ 2 School of Mechanical Engineering, Shandong University, Jinan 250061, China; melius@sdu.edu.cn \\ 3 Key Laboratory of High Efficiency and Clean Mechanical Manufacture of MOE/Key National, \\ Demonstration Center for Experimental Mechanical Engineering Education, Jinan 250061, China \\ 4 School of Mechatronics Engineering, Harbin Institute of Technology, Harbin 150001, China; \\ sunyzh@hit.edu.cn \\ * Correspondence: xichun.luo@strath.ac.uk; Tel.: +44-(0)-141-574-5280
}

Received: 7 February 2019; Accepted: 4 March 2019; Published: 7 March 2019

\begin{abstract}
Superhydrophobic surfaces have attracted extensive attention over the last few decades. It is mainly due to their capabilities of providing several interesting functions, such as self-cleaning, corrosion resistance, anti-icing and drag reduction. Nanosecond pulsed laser ablation is considered as a promising technique to fabricate superhydrophobic structures. Many pieces of research have proved that machined surface morphology has a significant effect on the hydrophobicity of a specimen. However, few quantitative investigations were conducted to identify effective process parameters and surface characterization parameters for laser-ablated microstructures which are sensitive to the hydrophobicity of the microstructured surface. This paper proposed and reveals for the first time, the concepts of process and product fingerprints for laser ablated superhydrophobic surface through experimental investigation and statistical analysis. The results of correlation analysis showed that a newly proposed dimensionless functional parameter in this paper, $R_{\text {hy }}$, i.e., the average ratio of $\mathrm{Rz}$ to Rsm is the most sensitive surface characterization parameter to the water contact angle of the specimen, which can be regarded as the product fingerprint. It also proposes another new process parameter, average laser pulse energy per unit area of the specimen $\left(I_{s}\right)$, as the best process fingerprint which can be used to control the product fingerprint $R_{h y}$. The threshold value of $R_{\text {hy }}$ and $I_{S}$ are 0.41 and $536 \mathrm{~J} / \mathrm{mm}^{2}$ respectively, which help to ensure the superhydrophobicity (contact angle larger than $150^{\circ}$ ) of the specimen in the laser ablation process. Therefore, the process and product fingerprints overcome the research challenge of the so-called inverse problem in manufacturing as they can be used to determine the required process parameters and surface topography according to the specification of superhydrophobicity.
\end{abstract}

Keywords: laser ablation; superhydrophobic surface; process fingerprint; product fingerprint; surface morphology

\section{Introduction}

Superhydrophobic surfaces are defined as those having a water contact angle larger than $150^{\circ}$ and sliding angle less than $10^{\circ}$. Artificial superhydrophobic surfaces, created by surface structuring or coating, have received tremendous attention in recent years. It is mainly due to their capabilities of providing several interesting functions, such as self-cleaning, corrosion resistance, anti-icing and drag reduction [1-6]. Surface chemical composition and morphology are two critical factors in determining their hydrophobicity [7-9]. The surface chemical composition affects the intrinsic contact angle, 
which can be measured by a liquid droplet deposited on a smooth surface. However, in artificial or natural materials, the maximum intrinsic contact angle is only approximately $120^{\circ}$ [8,9]. For this reason, more and more structuring technologies have been developed for the fabrication of superhydrophobic surfaces, including wet chemical reaction, lithography, rolling, 3D printing, micro milling and laser ablation $[2,6,10-15]$ etc.

Recently, laser ablation process has been demonstrated as a promising technique to fabricate superhydrophobic structures on varied materials, such as copper, aluminium, steel and glass [15-24]. Yang et al. investigated the wettability transition mechanism of laser ablated aluminium substrate, the results indicated that laser-ablated microstructures had the amplified effects on the hydrophobicity of the specimen [24]. Long et al. reported the effect of the laser pulse energy and width on the morphology of micro/nanostructures on a copper surface. They found that the morphology of the laser ablated structures is more sensitive to the laser pulse energy when nanosecond lasers with long pulse widths are used. Slightly decreasing the laser pulse energy results in the formation of no hierarchical micro- and nanostructures [17,20]. Gregorcic et al. fabricated a 316L stainless steel specimen with a pitch of $50 \mu \mathrm{m}$ at average pulse power of $0.6 \mathrm{~W}$ and $97 \%$ pulse overlapping rate and achieved a static contact angle of $153^{\circ}$ [18]. Long and Gregorcic both reported that variation of the pitch of channels resulted in completely different surface morphologies-from the highly porous surface to well-separated microchannels, which width and depth depend on laser fluence [18]. Duong Ta et al. concluded that surface roughness could be well controlled by laser power. The arithmetical mean height, Sa increased linearly when laser fluence was higher than $33 \mathrm{~J} / \mathrm{cm}^{2}$. The roughness was around 2 and 7 times larger than that of the untextured surface under fluences of 36 and $48 \mathrm{~J} / \mathrm{cm}^{2}$, respectively [23]. In addition, the effect of laser fluence and line separation on the contact angle of laser structured surfaces were investigated. Experimental results showed that the specimens possess superhydrophobicity has pitches of 50-150 $\mu \mathrm{m}$ and machined at the laser fluence of $36 \mathrm{~J} / \mathrm{cm}^{2}$ [23]. M. Conradi discovered that higher line density resulted in a higher contact angle. However, the average surface roughness Sa increased first then further decreased gradually with the increase of line density [19]. Thus, these researches have indicated that the laser machining parameters would significantly influence the hydrophobicity of the specimens while surface topography is a crucial factor to determine the superhydrophobicity of the specimen. However, there has been little systematic research exploring the correlation between surface topography and hydrophobicity of the specimen. Furtherly, the second challenge is to find out the most effective process parameter and surface characterization parameter for these microstructures which are sensitive to the hydrophobicity of the microstructured surface.

The identification of "product and process fingerprints" of laser ablated surface is a possible solution to solve the above issues. The concept of "product fingerprint" refers to those unique measurable characteristics (e.g., surface characterization parameters) on the laser ablated specimen that, if kept under control and within specifications, will ensure that the specimen possesses superhydrophobicity as required. The product fingerprint must be also sensitive to the variation of process parameters, hence it can be well-controlled by process parameters. For laser ablation process, since the surface characterization parameters are highly related to laser machining parameters, the "Process fingerprint" is defined as a specific process parameter to be controlled in order to maintain the manufacture of the specimen within the specified surface characterization parameters. The product and process fingerprints can be used as an objective function within an optimization tool to assist to determine the required surface topography and process parameters for the superhydrophobic surface.

The purpose of this paper is to reveal the product and process fingerprints for the laser ablation process of superhydrophobic surfaces on 316L stainless steel. A more generalized description can be achieved by linking laser machining parameters, surface characterization parameters and hydrophobicity of the specimen, which is beneficial to precise control of hydrophobicity and simultaneously enhancing its robustness. Therefore, product and process fingerprints are expected to provide a solution to the so-called inverse problem in manufacturing, which means the laser 
machining parameters and surface characterization parameters can be determined according to the required hydrophobicity, i.e., contact angle. Firstly, analysis of potential process and product fingerprint candidates will be carried out. Then, the most appropriate product fingerprint will be determined from values of Spearman and Kendall rank correlation coefficients according to the experimental results. Thirdly, a new process parameter will be put forward and chosen as the best process fingerprint. Lastly, the correlation between process fingerprint and functional performance, i.e., contact angle will be explored.

\section{Analysis of Process and Product Fingerprints}

Figure 1 illustrates the concept of process and product fingerprints in the laser ablation process for obtaining the superhydrophobic surface with an array of Gaussian holes of designed geometry. The comparison of all the potential candidates of process and product fingerprints will be discussed in detail later. Most research performed to date has focused on the correlation A; i.e., the effect of laser machining parameters on the contact angle of specimens. However, correlation A is actually composed of correlation B and C. Correlation B refers to the relationship between contact angle and product fingerprint, which is used to explain the underlying mechanism of effect of surface topography on hydrophobicity. Correlation $\mathrm{C}$ can describe the relationship between the process fingerprint and product fingerprint, to explore how the process parameters affect the surface topography. Thus, product fingerprint is a bridge to connect process parameters and functional performance-contact angle.

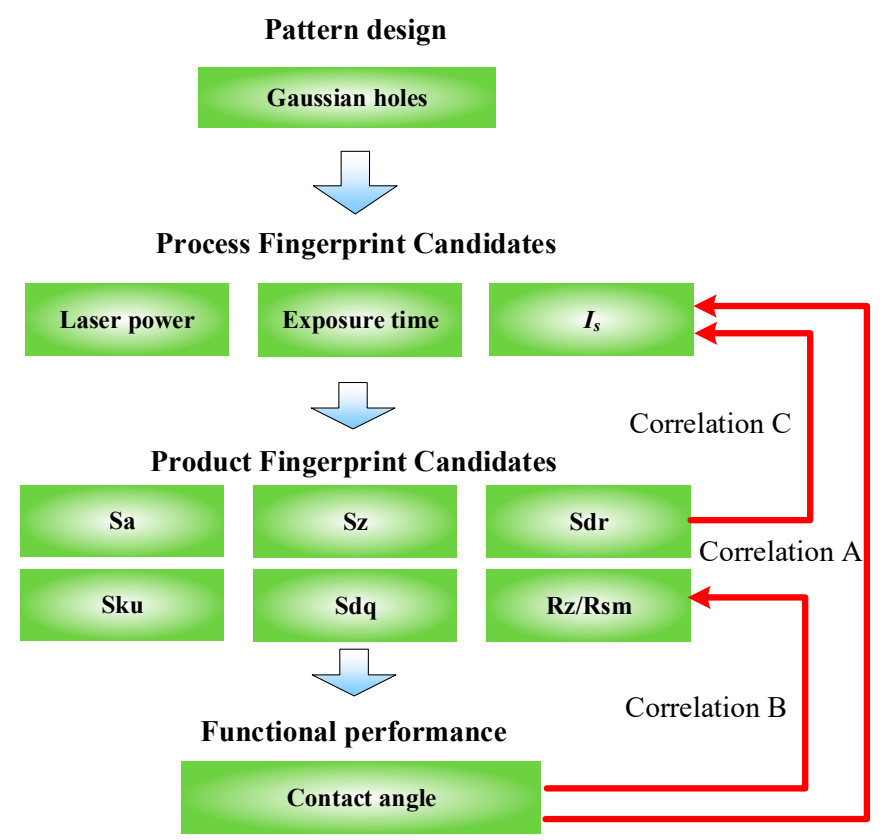

Figure 1. Concept of the process and product fingerprints in laser ablation of the superhydrophobic surface.

2.1. Analysis of Process Fingerprint Candidates: Laser Power, Exposure Time, Laser Pulse Energy Per Unit Area of Specimen

\subsubsection{Laser Power $(\mathrm{P})$}

In a nanosecond pulsed laser ablation process, the absorbed energy from the laser pulse melts the stainless steel and heats it to a temperature at which the atoms gain sufficient energy to enter into a gaseous state. Due to the vapour and plasma pressure, the molten materials are partially ejected from the cavity and form surface debris. At the end of a pulse, the heat quickly dissipates into the bulk of the work material and recast layer are formed. Therefore, laser power is a good candidate of process fingerprint as it determines the laser fluence which directly affects the formation of microstructures. The relationship between laser power, pulse repetition rate and peak power can be expressed as: 


$$
\begin{gathered}
E_{p}=\frac{P}{f_{p}} \\
P_{\text {peak }}=\frac{E_{p}}{\Delta \tau}
\end{gathered}
$$

where $P$ is laser average power, $f_{\mathrm{p}}$ is pulse repetition rate, $E_{p}$ is the energy of a single pulse, $P_{\text {peak }}$ is the peak power of laser and $\Delta \tau$ is the pulse duration, respectively.

\subsubsection{Exposure Time $(\mathrm{t})$}

For substrate with periodic Gaussian holes generated by the laser ablation process, the exposure time $t$ means the machining time for a single Gaussian hole, which determines the number of laser pulses that irradiated the surface. It has a significant effect on the dimension and morphology of Gaussian holes. As shown in Figure 2, the relationship between the number of irradiated pulse $\mathrm{N}$ and exposure time $t$ can be expressed as:

$$
\mathrm{N}=\frac{\mathrm{t}}{\mathrm{T}}
$$

where $\mathrm{T}$ is the pulse period.

Laser pulse energy per unit area of the specimen $I_{\mathrm{s}}$.

$I_{S}$ means the average laser pulse energy irradiated on a unit area of the specimen. This parameter depends on pulse repetition rate $f_{\mathrm{p}}$ and exposure time t. It can be expressed as:

$$
I_{s}=\frac{\mathrm{t} * f_{p} * E_{p}\left(\frac{\mathrm{L}}{\text { Pitch }}\right)^{2}}{\mathrm{~L}^{2}}
$$

According to Equation (1), $f_{p} * E_{p}=P$, hence Equation (4) can be simplified as:

$$
I_{s}=\frac{\mathrm{t} * P}{\mathrm{Pitch}^{2}}
$$

where pitch is the distance between adjacent Gaussian holes, and $\mathrm{L}$ is the length of the specimen.

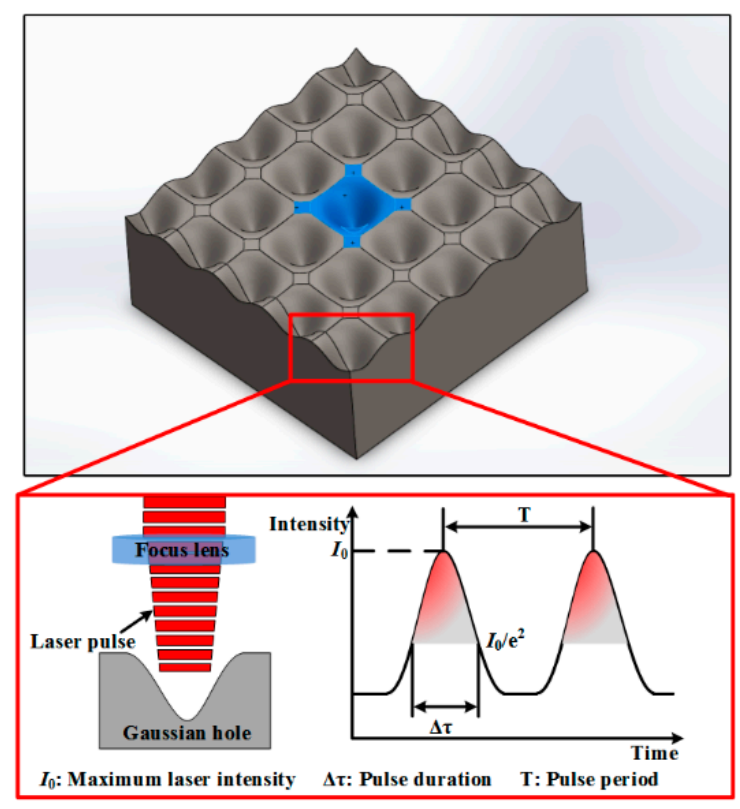

Figure 2. Schematic of periodic Gaussian holes machined by the laser ablation process. 


\subsection{Analysis of Product Fingerprint Candidates: $S a, S z, S k u, S d r, S d q, R_{h y}$}

In literature, two typical models have been developed to describe the behavior of a droplet on rough surfaces, i.e., the Wenzel and Cassie-Baxter models $[25,26]$. According to the Wenzel model, the droplet maintains contact with the structures and penetrates the asperities, and the surface contact area is increased. In addition, the contact angle $\theta_{w}$ can be described as:

$$
\begin{gathered}
\cos \theta_{w}=r \cos \theta \\
r=\frac{\text { actual surface area }}{\text { planar area }}
\end{gathered}
$$

where, $r$ is the roughness factor, which defined as the ratio of the actual area of the solid surface to the planar area. $\theta$ is the intrinsic contact angle of the material.

Alternatively, according to the Cassie-Baxter model, the droplet is not able to penetrate the microstructure spaces. However, in order to ensure the droplet cannot connect with the bottom of the microstructures, so the sag in height of water droplet between microstructures should be smaller than the depth of microstructures. Moreover, deep microstructures will help to form stable air pockets under the water droplet. Stable air pockets underneath the water droplet help the formation of superhydrophobicity with strong resistance against transition to the Wenzel state. Hence, sufficient depth of microstructure is essential to realize Cassie-Baxter state of the water droplet. The static contact angle $\theta_{C B}$ can be expressed as:

$$
\begin{gathered}
\cos \theta_{C B}=-1+f(1+\cos \theta) \\
f=\frac{\text { actual solid and liquid contact area }}{\text { planar area }}
\end{gathered}
$$

where $f$ is the fraction of the solid-liquid contact area.

The above analysis proves that the contact angles obtained in both Wenzel and Cassie-Baxter states are highly related to the vertical and horizontal feature of surface topography. Six surface characterization parameters that most probably correlated with the hydrophobicity of specimens are listed in Table 1. Sa, Sz and Sku are roughness parameters to characterize the height of the surface. $\mathrm{Sdr}, \mathrm{Sdq}, \mathrm{R}_{\mathrm{hy}}$ are hybrid parameters which determined from both height and horizontal parameters of the surface. For a rough surface, Sdr means the additional surface area contributed by the texture

\begin{tabular}{|c|c|c|}
\hline Name & Symbol & Meaning \\
\hline Arithmetical mean height & $\mathrm{Sa}$ & $\begin{array}{l}\text { The difference in height of each point compared to the arithmetical } \\
\text { mean of the surface. }\end{array}$ \\
\hline Maximum height & $\mathrm{Sz}$ & $\begin{array}{c}\text { The sum of the largest peak height value and the largest pit depth } \\
\text { value within the defined area. }\end{array}$ \\
\hline Kurtosis & Sku & $\begin{array}{c}\text { A measure of the sharpness of the roughness profile. } \\
\mathrm{Sku}<3 \text { : Height distribution is skewed above the mean plane. } \\
\mathrm{Sku}=3 \text { : Height distribution is normal. (Sharp portions and } \\
\text { indented portions co-exist.) }\end{array}$ \\
\hline & & Sku $>$ 3: Height distribution is spiked. \\
\hline Developed interfacial area ratio & $\mathrm{Sdr}$ & $\begin{array}{l}\text { The percentage of the definition area's additional surface area } \\
\text { contributed by the texture as compared to the planar definition area. }\end{array}$ \\
\hline Root mean square gradient & Sdq & $\begin{array}{l}\text { Root mean square of slopes at all points in the definition area. When } \\
\text { a surface has any slope, its Sdq value becomes larger. }\end{array}$ \\
\hline Average ratio of $\mathrm{Rz}$ to $\mathrm{Rsm}$ & $\mathrm{R}_{\text {hy }}$ & $\begin{array}{c}\text { Average ratio of the maximum height of profile }(\mathrm{Rz}) \text { and mean } \\
\text { width of the profile elements }(\mathrm{RSm})\end{array}$ \\
\hline
\end{tabular}
as compared to the planar definition area. Therefore, $1+\mathrm{Sdr}$ has the same meaning as the roughness factor $r$ in the Wenzel state.

Table 1. Product fingerprint candidates. 
Theoretical analysis proved that microstructures should have a high aspect ratio to provide a larger surface area and a smaller separation distance which will help to improve the stabilization of the solid-liquid-air composite interface [27]. However, present functional parameters cannot reflect the aspect ratio of surface asperities. Hence, $R_{h y}$ is proposed for the first time as a dimensionless functional parameter in this research and defined as the average ratio of Rz to Rsm. The subscript "hy" is the short abbreviation of hydrophobicity. The $R_{\text {hy }}$ is calculated from the average value of 60 lines that evenly distributed on the structured surface horizontally and vertically. A surface with large $R_{h y}$ can be obtained from a large Rz or smaller Rsm, which means the features of the surface should have a large depth or smaller separation distance (i.e., high density) in the horizontal direction.

\section{Experimental Details}

Laser machining experiments were carried out on AISI 316L stainless steel by varying the process parameters in order to identify the best product and process fingerprints. All the experiments were carried out on a hybrid ultra-precision machine, as shown in Figure 3. It is equipped with a nanosecond pulsed fiber laser which has a central emission wavelength of $1064 \mathrm{~nm}$. The laser source has a nominal average output power of $20 \mathrm{~W}$ and its maximum pulse repetition rate is $200 \mathrm{kHz}$. For a pulse repetition rate of $20 \mathrm{kHz}$, the average pulse duration is $100 \mathrm{~ns}$ and pulse energy is $1 \mathrm{~mJ}$. The laser machining parameters are listed in Tables 2 and 3. After the laser ablation process, the specimens were cleaned ultrasonically with deionized water, acetone and ethanol successively. Then the prepared specimens were silanized in a vacuum oven using silane reagent $(1 \mathrm{H}, 1 \mathrm{H}, 2 \mathrm{H}, 2 \mathrm{H}$-Perfluorooctyltriethoxysilane, $97 \%$, Alfa Aesar Ltd., Ward Hill, MA, USA), at $100{ }^{\circ} \mathrm{C}$ for $12 \mathrm{~h}$ to reduce their surface free energies.

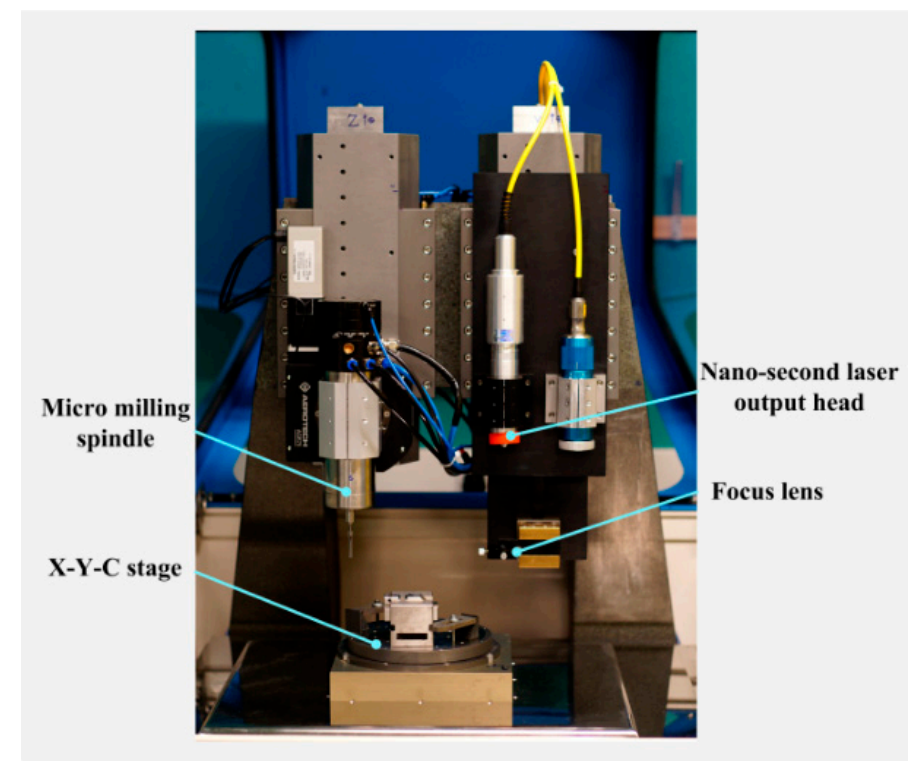

Figure 3. Experimental setup for laser machining trials.

Table 2. The laser machining parameters with varied laser power and pitch.

\begin{tabular}{cccccc}
\hline Pitch $(\boldsymbol{\mu m})$ & Laser Power $(\mathbf{W})$ & $\begin{array}{c}\text { Pulse Repetition } \\
\text { Rate }\end{array}$ & $\begin{array}{c}\text { Feed Rate } \\
(\mathbf{m m} / \mathbf{m i n})\end{array}$ & Exposure Time (s) & Pattern Types \\
\hline 90 & $4,6,10,14,20$ & $100 \mathrm{~K}$ & 200 & 0.4 & Gaussian holes \\
110 & $4,6,10,14,20$ & $100 \mathrm{~K}$ & 200 & 0.4 & Gaussian holes \\
130 & $4,6,10,14,20$ & $100 \mathrm{~K}$ & 200 & 0.4 & Gaussian holes \\
150 & $4,6,10,14,20$ & $100 \mathrm{~K}$ & 200 & 0.4 & Gaussian holes \\
\hline
\end{tabular}


Table 3. The laser machining parameters with varied exposure time and pitch.

\begin{tabular}{cccccc}
\hline Pitch $(\boldsymbol{\mu m})$ & Laser Power $(\mathbf{W})$ & $\begin{array}{c}\text { Pulse Repetition } \\
\text { Rate }\end{array}$ & $\begin{array}{c}\text { Feed Rate } \\
(\mathbf{m m} / \mathbf{m i n})\end{array}$ & Exposure Time (s) & Pattern Types \\
\hline 70 & 20 & $100 \mathrm{~K}$ & 200 & $0.2,0.4,0.6,1$ & Gaussian holes \\
90 & 20 & $100 \mathrm{~K}$ & 200 & $0.2,0.4,0.6,1$ & Gaussian holes \\
110 & 20 & $100 \mathrm{~K}$ & 200 & $0.2,0.4,0.6,1$ & Gaussian holes \\
130 & 20 & $100 \mathrm{~K}$ & 200 & $0.2,0.4,0.6,1$ & Gaussian holes \\
150 & 20 & $100 \mathrm{~K}$ & 200 & $0.2,0.4,0.6,1$ & Gaussian holes \\
\hline
\end{tabular}

The surface topography and varied surface characterization parameters of the laser structured surface were measured by a 3D laser scanning confocal microscope (VK-250, Keyence Corporation, Osaka, Japan). The static contact angle on surfaces was measured by a drop shape analyzer (Kruss Ltd., Hamburg, Germany). The selected water droplet volume was $5 \mu \mathrm{L}$. For each specimen, the contact angle of the water droplet was measured three times and the average value was adopted.

\section{Results and Discussion}

\subsection{Analysis of Product Fingerprint: $S a, S z, S k u, S d r, S d q, R_{h y}$}

The investigation of experimental results was carried out to identify the product fingerprint from six candidates related to surface topography. The product fingerprint is the indicator that has the highest level of correlation to contact angle. In this research, the Spearman rank correlation coefficient and Kendall rank correlation coefficient were employed to determine the product fingerprint. Spearman rank correlation coefficient evaluates how strong the correlation between two variables can be defined by a monotonic function. It measures the strength and direction of the monotonic association between two variables, a perfect Spearman correlation of +1 or -1 occurs when each variable is a perfect monotone function of the other [28]. A positive Spearman correlation coefficient corresponds to an increasing monotonic trend between two variables, while a negative value means a decreasing monotonic trend. In addition, Spearman rank correlation coefficient is appropriate for data that is not normally distributed. It can be used to identify a non-linear correlation between two variables. Kendall rank correlation coefficient is a statistic used to measure the ordinal association between two variables [29]. However, unlike the Spearman coefficient, Kendall rank correlation coefficient only considers directional agreement while does not consider the difference between ranks. Therefore, this coefficient is more appropriate for discrete data. This coefficient returns a value of -1 to 1 , where 0 is no correlation, 1 is a perfect positive correlation, and -1 is a perfect negative correlation. In most cases, the interpretations of Spearman and Kendall rank correlation coefficients are very similar and thus invariably lead to the same inferences. The above two coefficients were combined to determine the product fingerprint that has the maximum absolute value. The strength of the correlation between the variables can be evaluated by the absolute value of coefficients, as shown in Table 4.

Table 4. Interpretation of the strength of the correlation coefficient.

\begin{tabular}{cc}
\hline Value of Coefficient & Correlation Type \\
\hline 1 & Perfect correlation \\
$0.81-0.99$ & Strong correlation \\
$0.71-0.80$ & Good correlation \\
$0.51-0.70$ & Weak correlation \\
$0.01-0.50$ & Poor correlation \\
0 & No correlation \\
\hline
\end{tabular}

Figure 4 shows scatter plots between the contact angle and the six candidates of product fingerprint. With the increase of Sa, Sz, Sdr, Sdq and $\mathrm{R}_{\mathrm{hy}}$, the contact angle shows an increasing trend. It should be noted that a good linear relationship appears between $\mathrm{Sz}$ and contact angle, which 
is similar to the authors' previous study [15]. However, it can be observed that there is no apparent correlation between Sku and contact angle (Figure 4c). As shown in Figure 4d, increasing Sdr from 0.02 to 4.1 leads to contact angle increase rapidly from $89.5^{\circ}$ to $159^{\circ}$, but it has a minor impact on the contact angle when Sdr was further increased from 4.1 to 9.8. As Figure $4 \mathrm{f}$ indicates, the contact angle increases gradually from $89.5^{\circ}$ to $164^{\circ}$ with the value of $R_{\text {hy }}$ increasing from 0.06 to 0.94 .

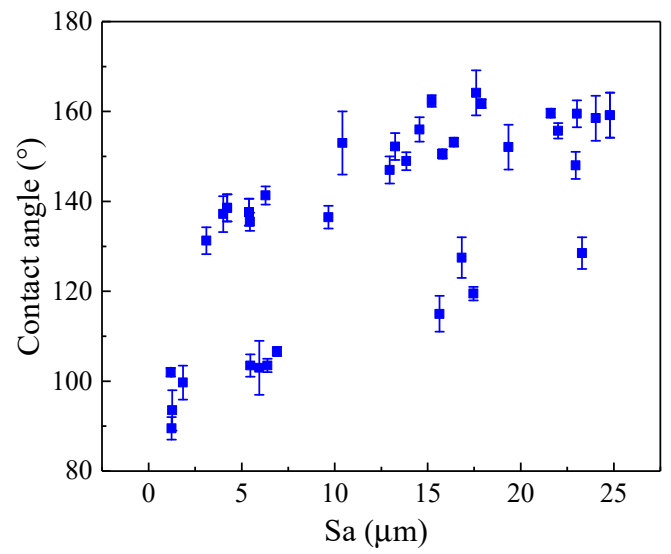

(a)

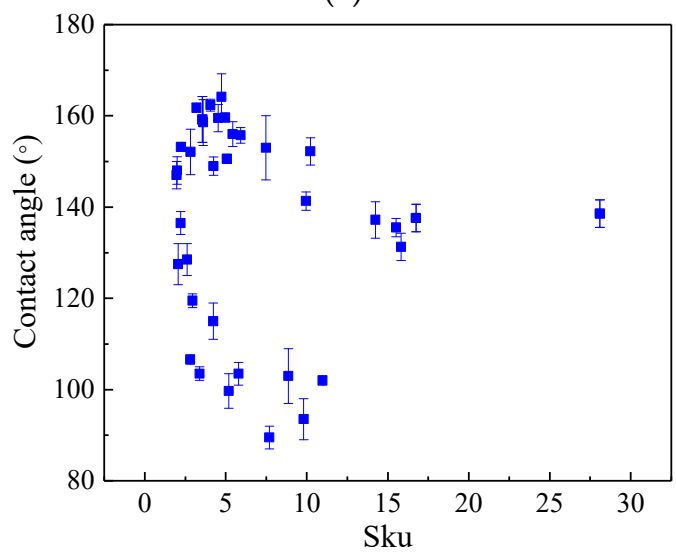

(c)

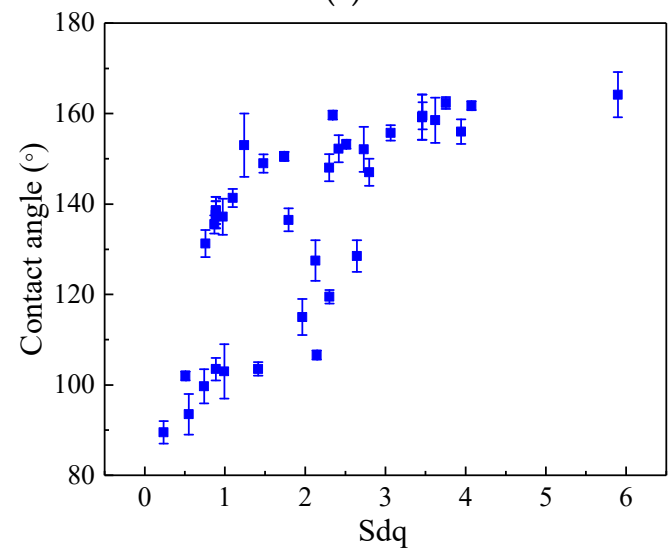

(e)

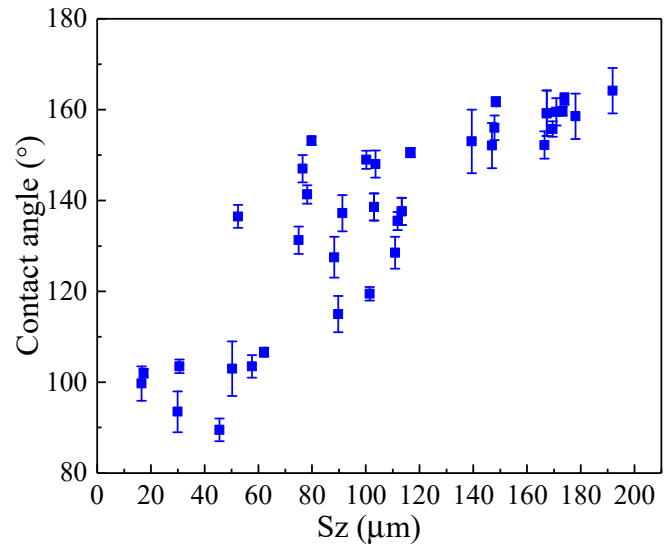

(b)

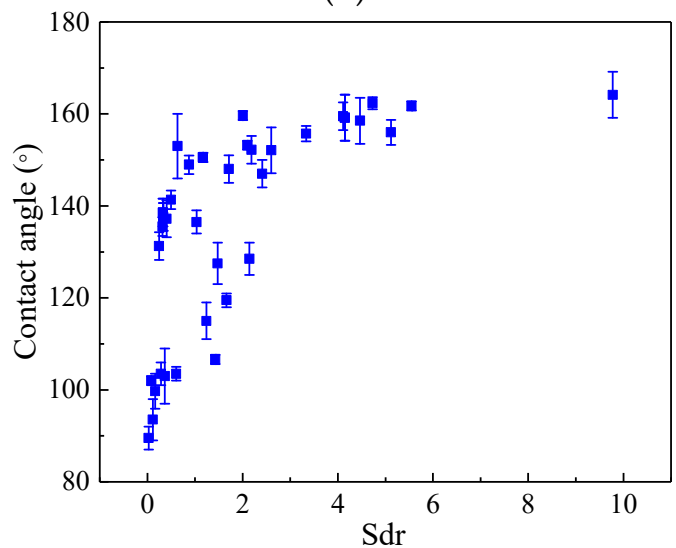

(d)

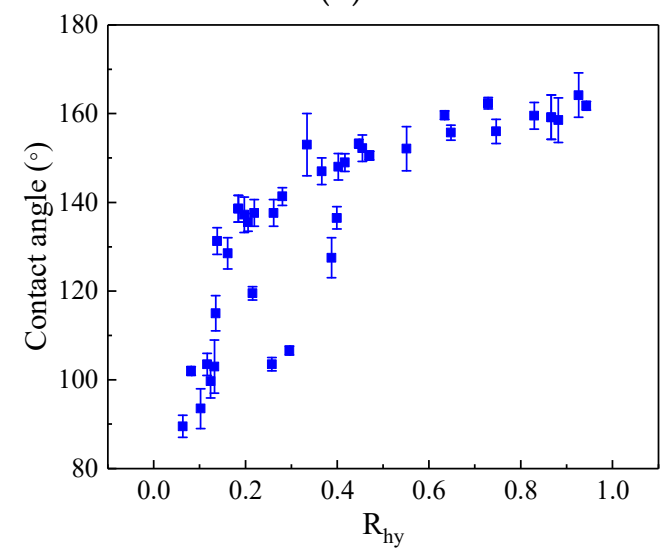

(f)

Figure 4. Influence of the product fingerprint candidates on the contact angle for Gaussian hole pattern: (a) Sa; (b) Sz; (c) Sku; (d) Sdr; (e) Sdq; (f) $\mathrm{R}_{\mathrm{hy}}$.

Figure 5 shows the variation of Spearman and Kendall rank correlation coefficient between contact angle and candidates of product fingerprint. According to the criterion in Table 4, Sz and $R_{\text {hy }}$ both have larger Spearman rank correlation coefficients with the contact angle, which are 0.89 and 0.92 
respectively. The Kendall rank correlation coefficient among Sz, $\mathrm{R}_{\text {hy }}$ and contact angle are 0.74 and 0.76. Thus, the results of Figure 5 suggest that $R_{\text {hy }}$ should be determined as the best product fingerprint as it has the maximum Spearman and Kendall rank correlation coefficients.

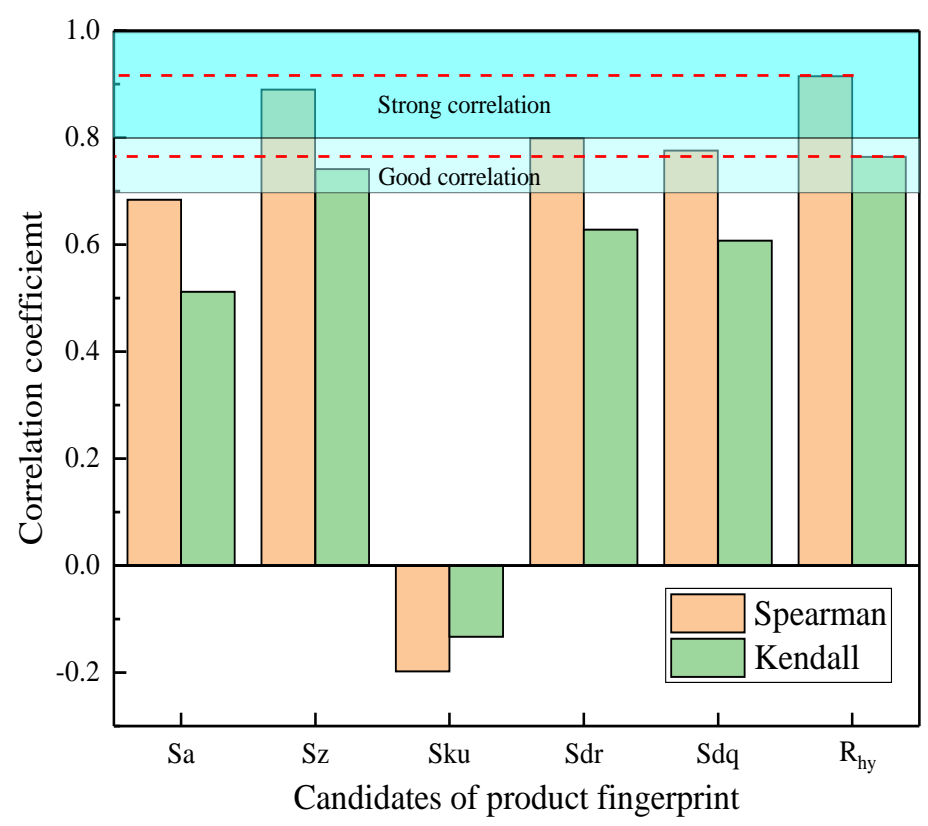

Figure 5. Spearman and Kendall rank correlation coefficient between the contact angle and six candidates of product fingerprint.

According to the results in Figure $4 \mathrm{f}$, an empirical equation was deduced to correlate the experimental $\mathrm{R}_{\text {hy }}$ and contact angle. The equation is expressed as:

$$
\theta_{A}=a-b * \mathrm{e}^{c * \mathrm{R}_{\mathrm{hy}}}
$$

where, $\theta_{A}$ is contact angle; $a, b$ and $c$ are constant values, equal to 164,105 and -4.9 respectively.

As shown in Figure $6 \mathrm{a}$, the regression curve has good precision to simulate the experimental data. We found that coefficient " $a$ " means the maximum contact angle ( $164^{\circ}$ in this research), the value of " $b$ " is equal to the initial contact angle $\left(105^{\circ}\right)$ of $316 \mathrm{~L}$ stainless steel after chemical modification. Thus, the contact angle of the specimen is highly related to its maximum contact angle, initial contact angle on a smooth surface and hydrophobicity functional parameter $R_{\text {hy }}$. According to Equation (10), the value of $R_{\text {hy }}$ is 0.41 when $\theta_{A}=150$. Thus, 0.41 can be regarded as the threshold value of $R_{\text {hy }}$ that ensure water contact angle of the specimen higher than $150^{\circ}$.

The dimensionless ratio $R_{h y}$ is the most sensitive candidate parameter for contact angle of the specimen, which can therefore, be regarded as product fingerprint. In literature, many studies proved that a high density of microstructures and smaller period of microstructure will help decrease solid-liquid contact area and increase its hydrophobicity $[22,30]$. With the increase of $R_{\text {hy }}$ from 0.138 to 0.943 (Figure 6b), Rsm decreased from $137.0 \mu \mathrm{m}$ to $81.8 \mu \mathrm{m}$. Therefore, the density of peaks shows a significant increasing trend. Moreover, the depth of microstructures shows an increasing trend, due to average Rz increased from $18.9 \mu \mathrm{m}$ to $77.2 \mu \mathrm{m}$. Therefore, it can be concluded that the superhydrophobicity will benefit from the increase of $R_{h y}$. 


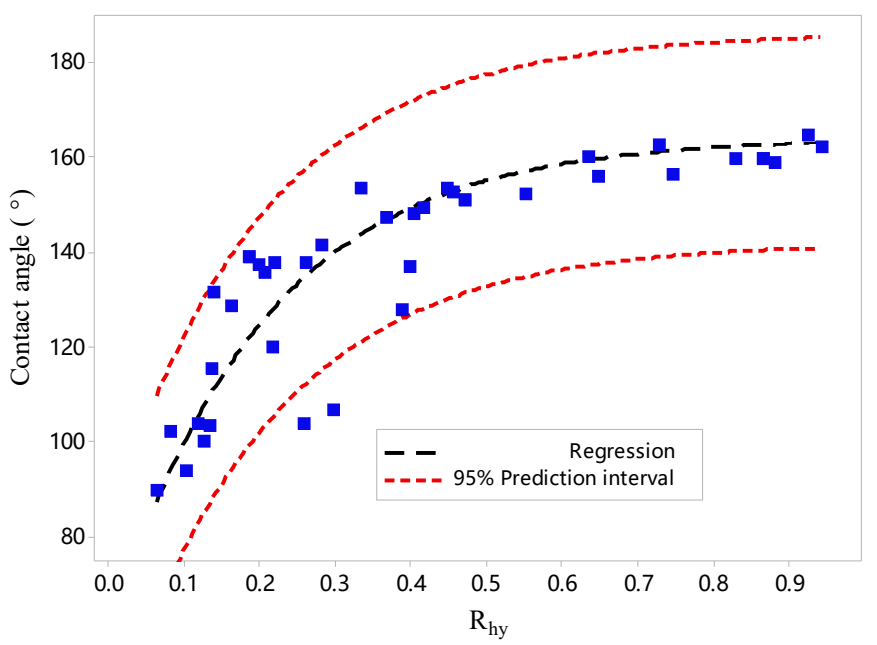

(a)
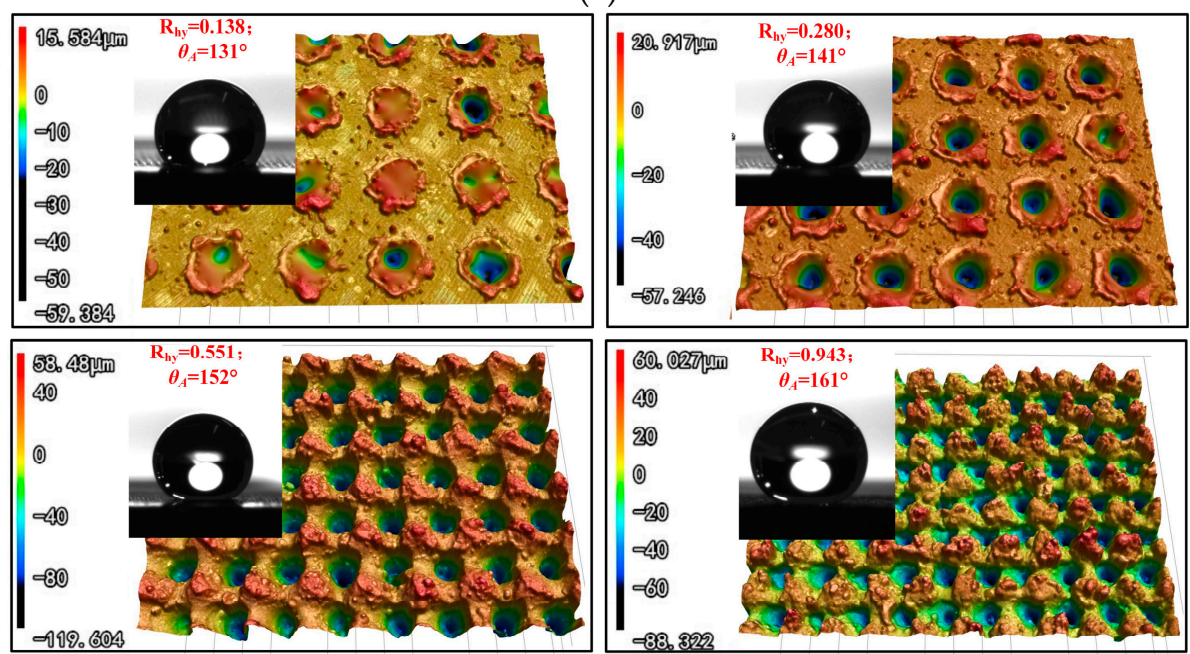

(b)

Figure 6. (a) Fitted line by exponential function between $R_{\text {hy }}$ and contact angle; (b) Surface morphology and shape of water drops on specimens with a different value of $R_{h y}$.

\subsection{Analysis of Process Fingerprints: $P, t$ and $I_{S}$}

The above section proves that $R_{\text {hy }}$ is the most appropriate product fingerprint to the laser ablated superhydrophobic structures on 316L stainless steel. In this section, further analysis of the experimental results will be performed to identify the best process fingerprint from the candidates $\mathrm{P}, \mathrm{t}$ and $I_{s}$, i.e., the process fingerprint which has the strongest correlation with $R_{h y}$. The control of process fingerprints helps to choose appropriate process parameter to obtain a surface with $R_{\text {hy }}$ greater than the threshold value $\left(R_{\text {hy }}>0.41\right)$. The correlation among laser power, pitch of Gaussian hole and $R_{\text {hy }}$ is shown in Figure 7. It shows that higher laser power and smaller pitch lead to a higher value of $R_{\text {hy }}$. Laser power and pitch of structures have combined effects on the value of $R_{h y}$. 


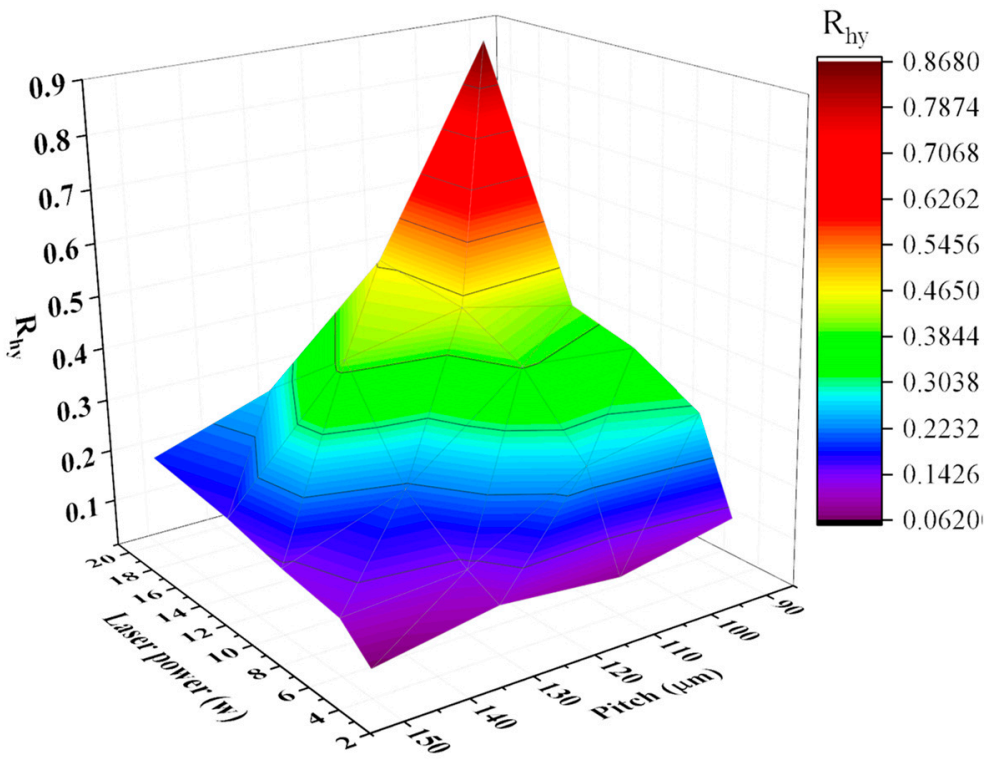

Figure 7. 3D colormap of the product fingerprint $\left(R_{h y}\right)$ as a function of laser power and pitch of Gaussian hole.

The effect of exposure time $t$ and pitch of Gaussian holes on the value of $R_{h y}$ is presented in Figure 8 . There is no significant linear correlation between exposure time and $R_{\text {hy }}$, but it does not mean exposure time has no effect on $R_{\text {hy }}$. As a whole, it can be found that the value of $R_{\text {hy }}$ shows a significant increasing trend with the reduction of pitch from $150 \mu \mathrm{m}$ to $70 \mu \mathrm{m}$.

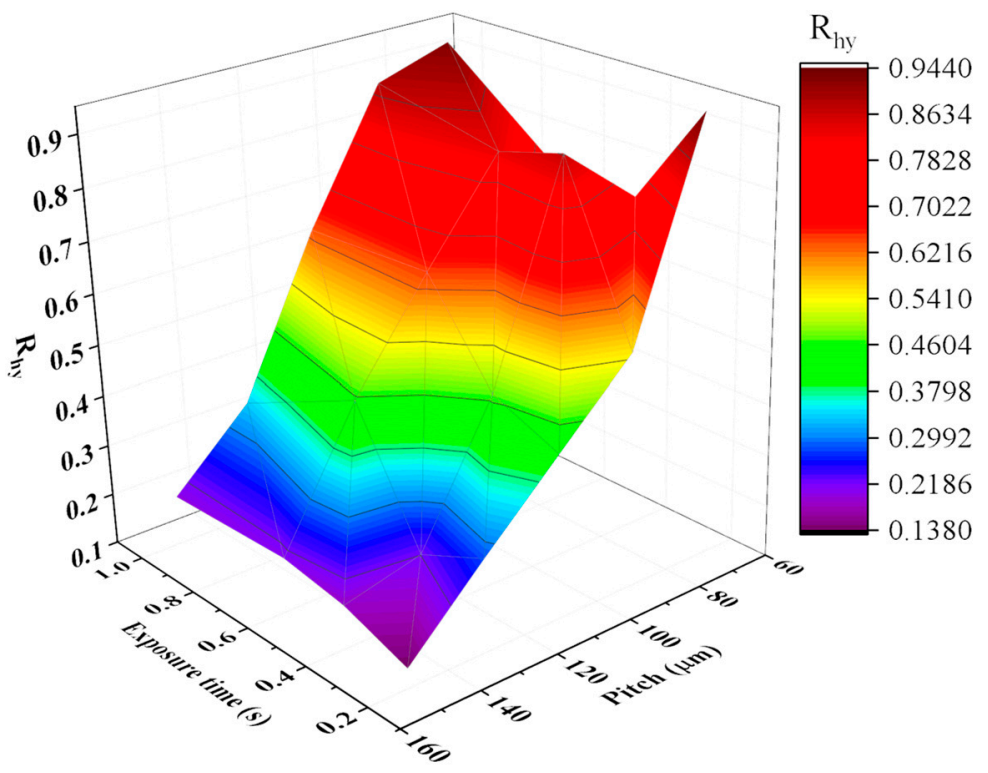

Figure 8. 3D colormap of the product fingerprint $\left(R_{\text {hy }}\right)$ as a function of exposure time and pitch of Gaussian holes.

The above analysis shows that laser power, pitch and exposure time have a collective influence on $R_{\text {hy }}$. Focusing one of them and ignoring the other two would lead to the determined correlation only effective in certain partial conditions. For instance, the $R_{\text {hy }}$ will increase with laser power, but only valid at a precondition of constant pitch and exposure time. Therefore, a comprehensive factor $I_{\mathrm{S}}$ was designed to represent the combined influence of laser power, pitch and exposure time. $I_{S}$ means the energy intensity that irradiated on the unit area of the specimen and can be calculated by the Equation (5). $I_{S}$ is proportional to the laser power $P$ and the exposure time $t$, but inversely proportional 
to the square of the pitch of the microstructures. Figure 9 reveals that the increasing $I_{s}$ leads $R_{\text {hy }}$ increase rapidly at first, and then level off to become asymptotic to the upper limit. The presence of upper limit means the further increased laser power, exposure time and smaller pitch cannot lead to a further increase of $R_{h y}$. The correlation between $I_{s}$ and $R_{h y}$ can be expressed as Equation (11). According to the calculation result, $I_{s}$ should be greater than $536 \mathrm{~J} / \mathrm{mm}^{2}$ to ensure $R_{\text {hy }}$ greater than 0.41 , hence the contact angle of the specimen will be larger than $150^{\circ}$.

$$
\mathrm{R}_{\mathrm{hy}}=0.895-0.898 * 0.9985^{I_{S}}
$$

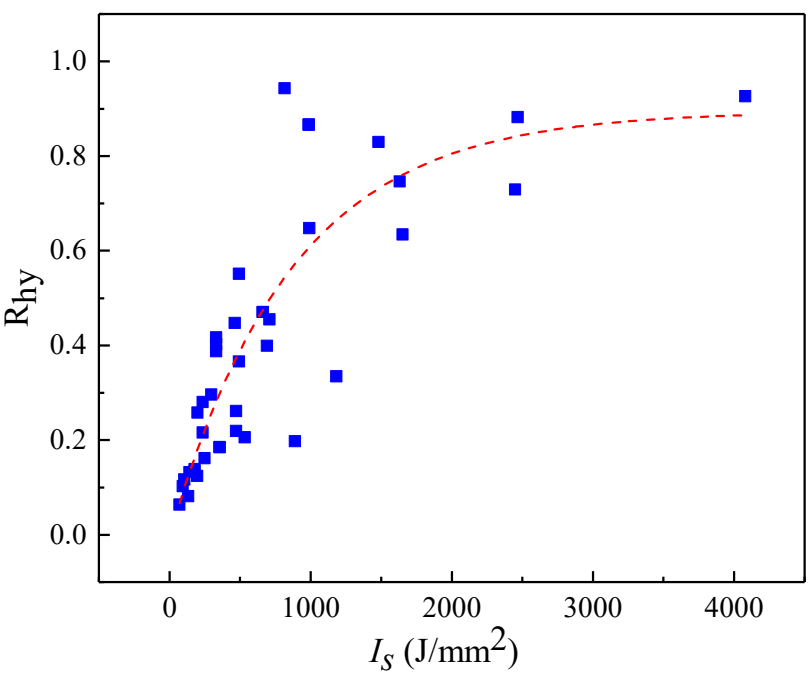

Figure 9. Scatter plots and fitted curve of $\mathrm{R}_{\mathrm{hy}}$ and $I_{s}$.

Therefore, the increased $I_{s}$ leads to rapidly increase of $R_{\text {hy }}$, the correlation between $R_{\text {hy }}$ can be described by the exponential function. $I_{s}$ is the most sensitive parameters among the investigated three process fingerprint candidates, so it is the best process fingerprint that can be used to control surface morphology, especially the product fingerprint $R_{h y}$.

\subsection{Correlation Between Laser Machining Parameters and Contact Angle}

As shown in Figure 10, 3D colormaps are used to display the relationship between laser power, exposure time, pitch of structures and contact angle. To sum up, the greater contact angle benefit from larger laser power and smaller pitch of microstructures except for some outliers.

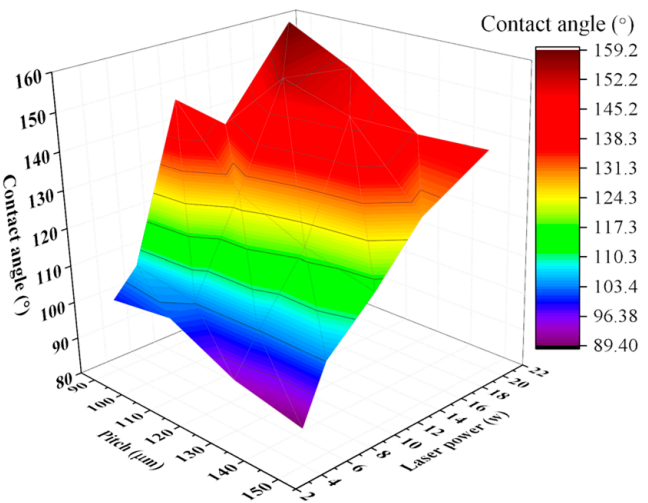

(a)

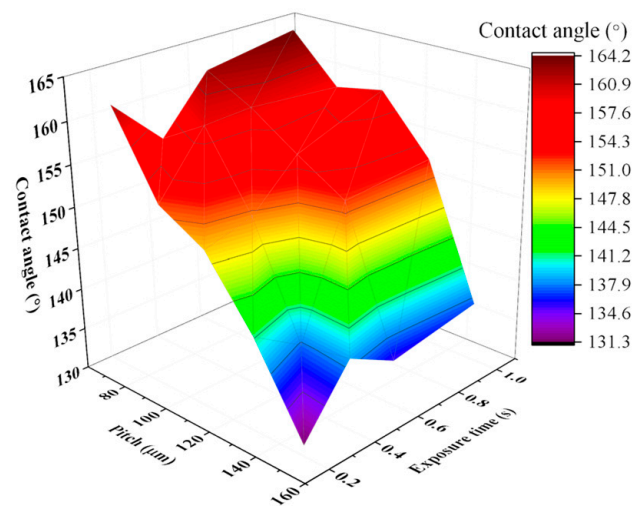

(b)

Figure 10. (a) 3D colormap of the contact angle as a function of laser power and pitch of microstructures; (b) 3D colormap of the contact angle as a function of exposure time and pitch of microstructures. 
Figure 11a shows the scatter diagram and fitted curve between contact angle and $I_{s}$. The increasing $I_{S}$ results in a rapid increase of contact angle at first, and then level off to become asymptotic to the upper limit when $I_{s}$ greater than $1000 \mathrm{~J} / \mathrm{mm}^{2}$. The empirical correlation between contact angle and $I_{S}$ can be expressed by Equation (12). When the value of $R_{\text {hy }}$ equals to the threshold value of 0.41 , the corresponding $I_{S}$ is $516.6 \mathrm{~J} / \mathrm{mm}^{2}$, which is very close to the value of $536 \mathrm{~J} / \mathrm{mm}^{2}$ obtain from Equation (11). Therefore, $I_{s}$ should be larger than $536 \mathrm{~J} / \mathrm{mm}^{2}$ in the laser ablation process, which help ensure the contact angle larger than $150^{\circ}$.

$$
\theta_{A}=a-b * \mathrm{e}^{d * I_{s}}
$$

where, $\theta_{A}$ is contact angle, $a=164, b=105, d=-0.0039$. Coefficients of $a$ and $b$ have the same meaning with Equation (10).

The surface morphology and shape of water drops on specimens with a different value of $I_{S}$ are shown in Figure 11b. With the increase of $I_{s}$, the depth and density of structures show a significant increasing trend. Thus, the surface topography and contact angle can be well controlled by choosing the appropriate process parameter $I_{s}$.

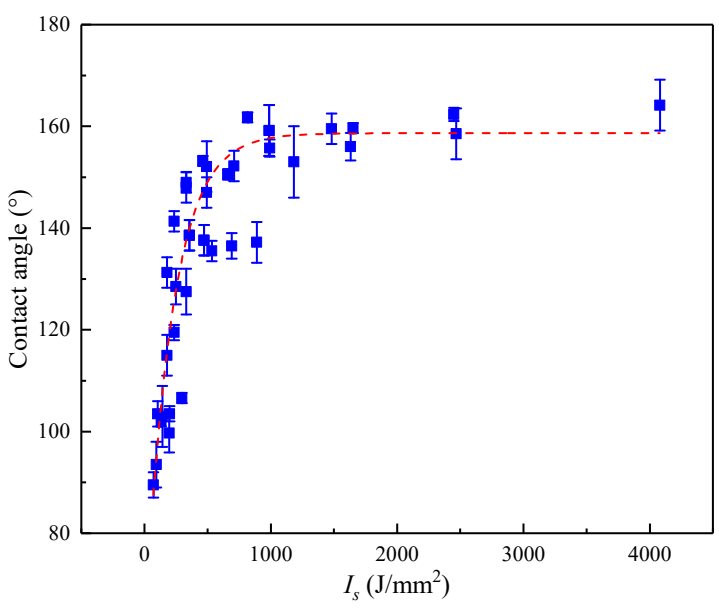

(a)

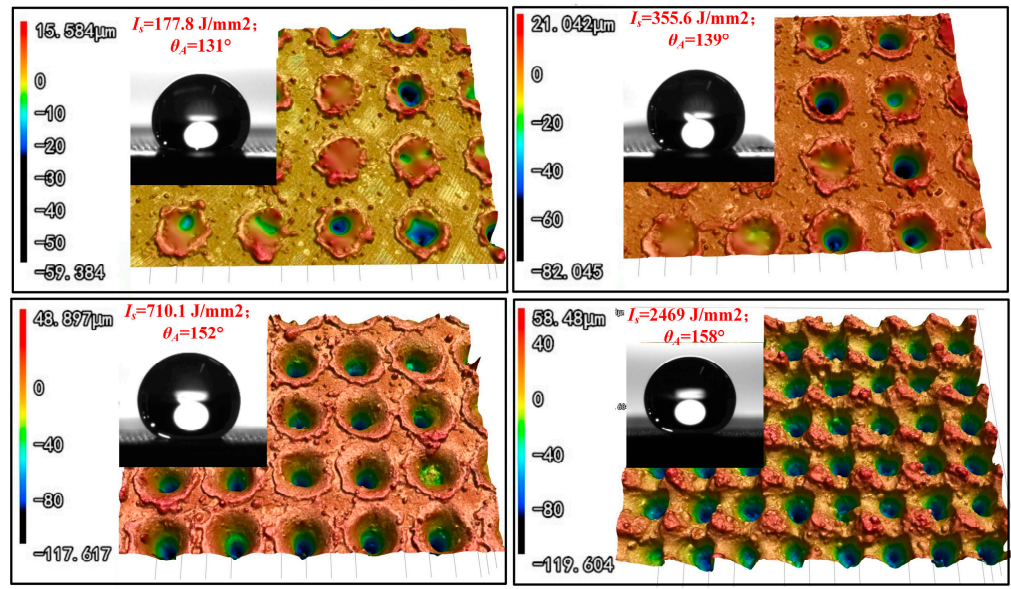

(b)

Figure 11. (a) Scatter plot and fitted curve between contact angle and $I_{s} ;(\mathbf{b})$ Surface morphology and shape of water drops on specimens with a different value of $I_{s}$.

\section{Conclusions}

In this study, the concepts of product and process fingerprint are put forward for the first time to reveal the correlations among process parameters, surface topography and functional performance, 
i.e., the contact angle of laser ablated superhydrophobic surface on 316L stainless steel. The most appropriate product fingerprint was determined by the indicators of Spearman and Kendall rank correlation coefficients. Then, the candidate that was most sensitive to product fingerprint was determined as the best process fingerprint. Lastly, the correlation between process fingerprint and functional performance was developed. The conclusions can be drawn as follows:

1 . The dimensionless surface functional characterization parameter $R_{h y}$, i.e., the average ratio of $\mathrm{Rz}$ to Rsm is the most sensitive parameter to contact angle of the specimen, which can be regarded as the product fingerprint.

2. Laser pulse energy per unit area on specimen $\left(I_{S}\right)$ represents the combining effect of laser power, exposure time and pitch of structure on surface topography. It is the best process fingerprint that can be used to control the product fingerprint $R_{h y}$.

3. The increasing $I_{S}$ leads to the value of $R_{\text {hy }}$ increase rapidly at first, and then level off to become asymptotic to the upper limit. A similar trend also can be found between $I_{S}$ - contact angle and $\mathrm{R}_{\text {hy }}$ - contact angle. The threshold value of $\mathrm{R}_{\text {hy }}$ and $I_{s}$ are 0.41 and $536 \mathrm{~J} / \mathrm{mm}^{2}$ respectively, which help to ensure the superhydrophobicity (contact angle larger than $150^{\circ}$ ) of the specimen in the laser ablation process.

Author Contributions: Conceptualization, Y.C., X.L. and Y.Q.; methodology, Y.C., Y.S.; writing—original draft preparation, Y.C.; writing - review and editing, X.L., Z.L., W.C. and Y.S.; supervision, X.L., Z.L. and Y.Q.; project administration, X.L. and Y.Q.

Acknowledgments: This research was undertaken in the context of MICROMAN project ("Process Fingerprint for Zero-defect Net-shape MICROMANufacturing", http://www.microman.mek.dtu.dk/). MICROMAN is a European Training Network supported by Horizon 2020, the EU Framework Program for Research and Innovation (Project ID: 674801). The authors would also gratefully acknowledge the financial support from the EPSRC (EP/K018345/1) and the International Cooperation Program of China (grant number 2015DFA70630) for this research.

Conflicts of Interest: The authors declare no conflict of interest.

Data Statement: All data underpinning this publication are openly available from the University of Strathclyde Knowledge Base at https: / / doi.org/10.15129/06ca19bc-e028-42b7-ad12-0279d57cc940.

\section{References}

1. Tong, W.; Xiong, D.; Wang, N.; Yan, C.; Tian, T. Green and timesaving fabrication of a superhydrophobic surface and its application to anti-icing, self-cleaning and oil-water separation. Surf. Coat. Technol. 2018, 352, 609-618. [CrossRef]

2. Li, S.; Page, K.; Sathasivam, S.; Heale, F.; He, G.; Lu, Y.; Lai, Y.; Chen, G.; Carmalt, C.J.; Parkin, I.P. Efficiently texturing hierarchical superhydrophobic fluoride-free translucent films by AACVD with excellent durability and self-cleaning ability. J. Mater. Chem. A 2018, 6, 17633-17641. [CrossRef]

3. Anitha, C.; Syed Azim, S.; Mayavan, S. Influence of particle size in fluorine free corrosion resistance superhydrophobic coating-Optimization and stabilization of interface by multiscale roughness. J. Alloys Compd. 2018, 765, 677-684. [CrossRef]

4. Rastegari, A.; Akhavan, R. The common mechanism of turbulent skin-friction drag reduction with superhydrophobic longitudinal microgrooves and riblets. J. Fluid Mech. 2018, 838, 68-104. [CrossRef]

5. Xie, X.; Weng, Q.; Luo, Z.; Long, J.; Wei, X. Thermal performance of the flat micro-heat pipe with the wettability gradient surface by laser fabrication. Int. J. Heat Mass Transf. 2018, 125, 658-669. [CrossRef]

6. Trdan, U.; Hočevar, M.; Gregorčič, P. Transition from superhydrophilic to superhydrophobic state of laser textured stainless steel surface and its effect on corrosion resistance. Corros. Sci. 2017, 123, 21-26. [CrossRef]

7. Karlsson, M.; Forsberg, P.; Nikolajeff, F. From hydrophilic to superhydrophobic: Fabrication of micrometer-sized nail-head-shaped pillars in diamond. Langmuir 2010, 26, 889-893. [CrossRef] [PubMed]

8. Nishino, T.; Meguro, M.; Nakamae, K.; Matsushita, M.; Ueda, Y. The Lowest Surface Free Energy Based on $-\mathrm{CF}_{3}$ Alignment. Langmuir 1999, 15, 4321-4323. [CrossRef]

9. Bell, M.S.; Shahraz, A.; Fichthorn, K.A.; Borhan, A. Effects of Hierarchical Surface Roughness on Droplet Contact Angle. Langmuir 2015, 31, 6752-6762. [CrossRef] [PubMed] 
10. Ta, D.V.; Dunn, A.; Wasley, T.J.; Kay, R.W.; Stringer, J.; Smith, P.J.; Connaughton, C.; Shephard, J.D. Nanosecond laser textured superhydrophobic metallic surfaces and their chemical sensing applications. Appl. Surf. Sci. 2015, 357, 248-254. [CrossRef]

11. Ngo, C.V.; Chun, D.M. Fast wettability transition from hydrophilic to superhydrophobic laser-textured stainless steel surfaces under low-temperature annealing. Appl. Surf. Sci. 2017, 409, 232-240. [CrossRef]

12. Ngo, C.V.; Chun, D.M. Effect of Heat Treatment Temperature on the Wettability Transition from Hydrophilic to Superhydrophobic on Laser-Ablated Metallic Surfaces. Adv. Eng. Mater. 2018, 20, 1-11. [CrossRef]

13. Kim, J.H.; Mirzaei, A.; Kim, H.W.; Kim, S.S. Facile fabrication of superhydrophobic surfaces from austenitic stainless steel (AISI 304) by chemical etching. Appl. Surf. Sci. 2018, 439, 598-604. [CrossRef]

14. Brinksmeier, E.; Reese, S.; Klink, A.; Langenhorst, L.; Lübben, T.; Meinke, M.; Meyer, D.; Riemer, O.; Sölter, J. Underlying Mechanisms for Developing Process Signatures in Manufacturing. Nanomanuf. Metrol. 2018, 1, 193-208. [CrossRef]

15. Cai, Y.; Chang, W.; Luo, X.; Sousa, A.M.L.; Lau, K.H.A.; Qin, Y. Superhydrophobic structures on 316L stainless steel surfaces machined by nanosecond pulsed laser. Precis. Eng. 2018, 52, 266-275. [CrossRef]

16. Yang, C.; Tartaglino, U.; Persson, B.N.J. Influence of surface roughness on superhydrophobicity. Phys. Rev. Lett. 2006, 97, 1-4. [CrossRef] [PubMed]

17. Long, J.; Cao, Z.; Lin, C.; Zhou, C.; He, Z.; Xie, X. Formation mechanism of hierarchical Micro- and nanostructures on copper induced by low-cost nanosecond lasers. Appl. Surf. Sci. 2019, 464, 412-421. [CrossRef]

18. Gregorčič, P.; Šetina-Batič, B.; Hočevar, M. Controlling the stainless steel surface wettability by nanosecond direct laser texturing at high fluences. Appl. Phys. A Mater. Sci. Process. 2017, 123, 1-8. [CrossRef]

19. Conradi, M.; Drnovšek, A.; Gregorčič, P. Wettability and friction control of a stainless steel surface by combining nanosecond laser texturing and adsorption of superhydrophobic nanosilica particles. Sci. Rep. 2018, 8, 2-10. [CrossRef] [PubMed]

20. Long, J.; He, Z.; Zhou, C.; Xie, X.; Cao, Z.; Zhou, P.; Zhu, Y.; Hong, W.; Zhou, Z. Hierarchical micro- and nanostructures induced by nanosecond laser on copper for superhydrophobicity, ultralow water adhesion and frost resistance. Mater. Des. 2018, 155, 185-193. [CrossRef]

21. Ngo, C.V.; Chun, D.M. Control of laser-ablated aluminum surface wettability to superhydrophobic or superhydrophilic through simple heat treatment or water boiling post-processing. Appl. Surf. Sci. 2018, 435, 974-982. [CrossRef]

22. Park, K.C.; Choi, H.J.; Chang, C.H.; Cohen, R.E.; McKinley, G.H.; Barbastathis, G. Nanotextured silica surfaces with robust superhydrophobicity and omnidirectional broadband supertransmissivity. ACS Nano 2012, 6, 3789-3799. [CrossRef] [PubMed]

23. Ta, V.D.; Dunn, A.; Wasley, T.J.; Li, J.; Kay, R.W.; Stringer, J.; Smith, P.J.; Esenturk, E.; Connaughton, C.; Shephard, J.D. Laser textured superhydrophobic surfaces and their applications for homogeneous spot deposition. Appl. Surf. Sci. 2016, 365, 153-159. [CrossRef]

24. Yang, Z.; Liu, X.; Tian, Y. Insights into the wettability transition of nanosecond laser ablated surface under ambient air exposure. J. Colloid Interface Sci. 2019, 533, 268-277. [CrossRef] [PubMed]

25. Cassie, B.D. Wettability of porous surfaces. Trans. Faraday Soc. 1944, 40, 546-551. [CrossRef]

26. Wenzel, R.N. Resistance of Solid Surfaces To Wetting By Water. Ind. Eng. Chem. 1936, 28, 988-994. [CrossRef]

27. Nosonovsky, M.; Bhushan, B. Roughness optimization for biomimetic superhydrophobic surfaces. Microsyst. Technol. 2005, 11, 535-549. [CrossRef]

28. Spearman's Rank Correlation Coefficient. Available online: https://en.wikipedia.org/wiki/Spearman\% 27s_rank_correlation_coefficient (accessed on 21 January 2019).

29. Kendall Rank Correlation Coefficient. Available online: https://en.wikipedia.org/wiki/Kendall_rank_ correlation_coefficient (accessed on 21 January 2019).

30. Celia, E.; Darmanin, T.; Taffin de Givenchy, E.; Amigoni, S.; Guittard, F. Recent advances in designing superhydrophobic surfaces. J. Colloid Interface Sci. 2013, 402, 1-18. [CrossRef] [PubMed]

(C) 2019 by the authors. Licensee MDPI, Basel, Switzerland. This article is an open access article distributed under the terms and conditions of the Creative Commons Attribution (CC BY) license (http:// creativecommons.org/licenses/by/4.0/). 